Review Article

\title{
Nanomedicine and prospect of medical science
}

\section{What is Nanotechnology?.'}

\section{Definition}

"Nanotechnology is the art and science of manipulating matter at the nanoscale" that implies range of technologies, techniques and processes that changes maneuvering of matter at smallest scale (from 1 to $100 \mathrm{~nm}^{2}$ ) where unique phenomena of particles enable novel applications. National Science and Technology Council (USA) claim that "Nanotechnology is an enabling technology that will change the nature of almost every human-made object in the next century."

\section{Nano scale ${ }^{2}$}

$$
\begin{aligned}
& \text { Nanometer }=1 \times 10^{-9} \text { meter } \\
& =1 \times 10^{-3} \mu \mathrm{m} \\
& =3.281 \times 10^{-9} \mathrm{ft} \\
& =39.37 \times 10^{-9} \text { inchs }
\end{aligned}
$$

Equivalence of a nanometer. ${ }^{2-4}$

a. There are 1 billion nanometers in 1 meter

b. There are 1 million micrometers (or microns) in 1 meter

c. A line of ten $\mathrm{H}^{+}$atoms lined up side by side is 1 nanometer long

d. Finger nail grows 1 nanometer in 1 second

e. The diameter of our hair is about 50,000 nanometers

f. Abbreviation for nanometer is " $\mathrm{nm}$ "

\section{How small is nano ${ }^{5}$}

An elementary school child is 1 meter tall $(1$ meter $=100 \mathrm{~cm})$

a. One hand is 10 time smaller than child equal to $10 \mathrm{~cm}$

b. One finger breath is 10 time smaller than hand equal to 1 $\mathrm{cm}=10 \mathrm{~mm}$

c. One freckle is 10 time smaller than finger breath equal to $1 \mathrm{~mm}=1000 \mu \mathrm{m}$

d. One strand of hair is 10 time small than freckle equal to $100 \mu \mathrm{m}$

e. One $\mathrm{RBC}$ is 10 time smaller than hair equal to $10 \mu \mathrm{m}$

f. One bacteria is 10 time smaller than $R B C$ equal to $1 \mu \mathrm{m}=1000 \mathrm{~nm}$

g. One virus is 10 time smaller than bacteria equal to $100 \mathrm{~nm}$

h. Cell membrane is 10 time smaller than virus equal to $10 \mathrm{~nm}$

i. A sugar molecule is 10 times smaller than cell membrane equal to $1 \mathrm{~nm}$ wide!

j. An atom is 10 times smaller than sugar molecule $1 \mathrm{~nm}$ !

Materials found in nature are typically referenced to have the following dimensions. ${ }^{6}$

1. Atom $0.1 \mathrm{~nm}$

\author{
Volume 2 Issue 3 - 2015
}

Lt Colonel Abul Kalam Azad

Department of anesthesiology and intensive care, Combined Military Hospital, Bangladesh

Correspondence: Lt Colonel Abul Kalam Azad, Classified anesthesiologist, Department of anesthesiology and intensive care, Combined Military Hospital, Dhaka-1206, Bangladesh, Tel 008801715010956,Email azad100864@gmail.com

Received: February 15, 20I5 | Published: April 10, 2015
2. DNA (width) $2.5 \mathrm{~nm}$

3. Protein $5-50 \mathrm{~nm}$

4. Virus $5-100 \mathrm{~nm}$

5. Bacteria $1,000-10,000 \mathrm{~nm}$

6. White Blood Cell 10,000nm

\section{What makes Nano different? ${ }^{7-9}$}

There are significant differences in the properties of the particles between visible scale and optical scale. When particles are constituted with dimensions of $1-100 \mathrm{~nm}$, the properties of materials change significantly in comparison to larger scale. At this scale, behavior and properties of particles follow quantum physical rule. In nanoscale properties of materials are size dependent like melting point, fluorescence, electrical conductivity, magnetic permeability, and chemical reactivity which change as a function of the size of the particle. Nanotechnology is not merely functioning at ever smaller dimensions but functioning at the nanoscale enabling scientists to exploit the distinct physical, chemical, mechanical, and optical properties of materials that naturally occur at that scale.

Following are the two reasons why the characteristics of material can be different on a nanoscale. First, nanoparticles having relatively larger surface area than that of particles at larger surface can initiate particles chemically more reactive inert materials become reactive at nonoscale and having impact their electrical, magnetic and optical properties. Secondly particles less than $50 \mathrm{~nm}$, the laws of classical physics give way to quantum effects, provoking optical, electrical and magnetic behavior different form those of the same marerial at a larger scale.

\section{Quantum bio-physical activities in living organisms ${ }^{10}$}

There is very limited information about quantum molecular activities in living organisms. Indeed. biologists recognize the necessity of accepting operational harmonization of dynamic metabolic 
activities of life and spoke largely about the communication of wellspecified "signals" between DNA/RNA and molecular proteins. So to say, biologist's upto now did not accept the challenge of providing physical illustration of such signals, so their scientific findings remain very limited. Therefore, there remains an open question for physical illustration of space/time harmonious biological metabolic activities of biological signals to get more complete understanding in the study of living organism.

\section{Nanomedicine"I}

Nanomedicine is defined as the repairing, constructing, monitoring and controlling of human biological systems at the molecular level, by applying engineered nanodevices, nanorobots and nanostructures. In other way, the detection and controlled manipulation of human biological system at the molecular level are accomplished through engineered nanodevices, nanorobots and/or nanostructures.

\section{Necessity of knowing molecular nanotechnology ${ }^{12}$}

The body is incessantly under threats from the environmental hazards and troubles; the immune system is constantly confronting a silent war against toxins, bacteria, fungi, parasites and viruses hence they are perpetually attacking the body and trying to cause harm.

\section{Applications as diagnostic purposes ${ }^{13}$}

Biomedical laboratory diagnosis plays a key role in today's health care. Most testing is done on a macroscopic scale, size reduction of biomedical lab tests has several advantages: not only does it lead to a marked reduction of the sample volume needed for testing, but it also results in a marked reduction of (potentially expensive) reagents. Last but not least, it may lead to a significant reduction in the time required.

Nanotechnology could significantly improve diagnostic capabilities. Nanomedicine will increase the efficiency and accuracy of diagnosis from samples of body fluids. For example, some companies are attempting to develop microchips that use electrodes to identify the dielectric properties of cancerous cells, viruses, and bacteria in body fluids. Nanomedicine could result in noninvasive devices that can enter the body to determine glucose levels, distinguish between normal and cancerous tissues, and provide genetic screening for multiple diseases.

\section{Applications as monitoring purposes}

Nanomonitoring devices continuously monitor the patient's physical condition and will construct a detailed model of the patient's body thereby formulate a predictive approach to cure the disease along with other ailments. Gathering information sensors/nanocomputers compute and analyses various potential evidence based treatment for suggestions, even in routine diagnosis. Monitoring devices could make it possible to identify problems earlier thereby enabling more striking and holistic treatment approach.

\section{Applications as therapeutic purposes}

The prospect of applications of nanotechnology in medicine are highly promising in disease diagnosis, drug delivery targeted at specific sites in the body as well as molecular imaging since there are being extensively researched and under clinical trials. The concerned over a period of time regarding deleterious consequences of systemic biodistribution include toxicity to non-target tissues, difficulty in maintaining drug concentrations within therapeutic windows, and metabolism and excretion of drugs, all of which can reduce efficacy. Drug delivery systems based on nanotechnology can properly pacify these problems by balancing requirements and demands targeting tissues within therapeutic action. Multifunctional nano-delivery systems can also have multidimensional task of targeting, diagnostic, and therapeutic approach like delivering medication to the exact location, killing of bacteria, viruses \& repairing damaged cancer cells as well as tissue perfusion by transporting oxygen.

\section{Applications as medical robotics and nanodevices ${ }^{14}$}

Applications of nanorobotic early diagnosis and targeted drug delivery for cancer, biomedical instrumentation, surgery, Future medical nanotechnology are desired to employ nanorobots injected into the patients for repairing or detecting damages and infections at cellular level. Upon completion of their mission nanorobots are expected to exfuse themselves through human excretory system. Some of the most critical challenges for such an initiative include finding a means to power the device, finding a means to control the device, and ensuring that the device does not injure the patient in some unanticipated way.

\section{Application of nanotechnology in anesthesia, pain and critical care $^{15}$}

\section{General anesthesia}

At present, while providing general anaesthesia (GA) anaesthesiologists assessing depth of anaesthesia by certain clinical parameters such as pulse, respiration, systolic blood pressure, tearing and colour of blood when sophisticated monitoring is not available. Nanodevices enabled electronic chips to be implanted and linked to the human nervous system. Thereby it would allow to detect and control nerve impulses to maintain depth of anaesthesia analyzed by an external computer.

Regional anesthesia: Over dose of local anaesthetic create hazards anaesthesiologists as well as for patients as there are no antidotes available. By virtue of nanotechnology, an antidote of local anesthetic overdose is possible.

Pain medicine: Pain and palliative care are upcoming superspeciality of anaesthesiology. Narcotics are commonly used drugs for chronic pain which cause significant addiction. To overcome this, sustained release anaesthetics have been tried in the past, but due to toxicity this has become unsuccessful. Use of saxitoxin, formulated by utilizing nanotcechlogy revolutionize the treatment of chronic and even acute pain. It is said that, saxitoxin is a potent anaesthetic, bundled with liposomes which is slow- release formulation, nontoxic to the nerves or the surrounding tissues produce nerve block lasting from days to week's even months.

Critical care: Critical care medicine is under the domain of anaesthesiologists worldwide. But infection control, ventilator dependence, antibiotic resistance is the prime concern of ICU. Overcoming those critical problems nanotechnology is showing the hope to find a solution to those problems. Related revolutionize inventions are vasculoids, respirocytes and clottocytes.

"Respirocyte" is expected to be able to deliver more oxygen to the tissues than natural red blood cells and can manage carbonic acidity. Primary medical applications of respirocytes would include transfusable blood substitution; partial treatment for anemia, lung disorders, enhancement of cardiovascular/neurovascular procedures, tumour therapies and diagnostics, prevention of asphyxia, artificial breathing, and a variety of sports, veterinary and battlefield. Multifunctional nano-delivery systems could transport oxygen to tissue. 
Nanomedicine is concerned with the possible medical applications of nanotechnology, such as the use of nano materials for clinical purposes, as well as applications such as the development of nanosized biosensors, nanosized drug delivery systems (e.g. using polymerbased nanoparticles), and molecular nanotechnology approaches to detecting and treating disease. Current problems for nanomedicine also involve understanding the toxicity and environmental impact of nanoscale materials, as well as the use of nanotechnology to improve diagnostic imaging.

\section{Application of nanomedicine in medical practice ${ }^{16}$}

Nano biotechnology has applications in practically every branch of medicine and surgery. Nano biotechnology forms the basis of many new devices being developed for medicine and surgery like nanorobots. It has applications in practically every branch of medicine and surgery for instance medicine concern with cancer (nano oncology), neurological disorders (nano neurology), cardiovascular disorders (nano cardiology), diseases of bones and joints (nano orthopedics), diseases of the eye (nano ophthalmology), likewise surgery concern with vascular surgery by nanorobots introduced into the vascular system, tissue engineering with nano biotechnology scaffolds, implantation of rejection-resistant artificial tissues and organs, remote controlled nanorobots for detection and destruction of cancer, nano sensors implanted in catheters to provide real-time data during surgery, nano laser surgery and infectious diseases as well. Nanomedicine will also improve biological therapies such as vaccination, cell therapy and gene therapy.

\section{Disadvantages/challenges of nanomedicine and technology ${ }^{17}$}

i. Bioethical researchers believe that nanomedicine could be manipulated to harm the human body rather than healing it.

ii. What would be the use of technology that can't be seen or regulated? How efficient and effective will be the guiding system, which can direct the devices/drugs in the right organ sparing the wrong one?

iii. What would happen if the nanomedicine technology is used for terrorism purposes? Particles that can't be seen or easily controlled would enter the body and deliver harmful substances such as toxins.

iv. The most dangerous nano-application use for military purposes is the Nano- bomb that contains engineered self-multiplying deadly viruses that can continue to wipe out a community, country or even a civilization.

v. Nanobots because of their replicating behavior can be big threat for GRAY GOO

vi. Is there any assurance for the nano-medicinal technologies to be non-toxic and eco-friendly?

vii. Will it be practical, implementable and affordable?

\section{Conclusion ${ }^{12,14}$}

As Richard E Smalley, PhD, 1996 Nobel Laureate, once said, "Human health has always been determined on the nanometer scale; this is where the structure and properties of the machines of life work in every one of the cells in every living thing. The practical impact of nano science on human health will be huge." Nanotechnology will help to develop health by enriching the efficacy and safety of nano systems and nanodevices. Moreover, early diagnosis, implants with improved properties, cancer treatment and minimum invasive treatment for heart disease, diabetes and other diseases are anticipated. In future, nanotechnology will play a pivotal role in developing nanomedicine which would revolutionize opportunities for early disease detection, diagnostic and therapeutic procedures to improving health and enhancing physical abilities, and ensuring precise and effective therapy to the patients. Nanomedicine, though it is in its infancy expect to have prospective change in medical research dramatically in the 21 st century. Nano medical devices are expected to apply for analytical, imaging, detection, diagnostic and therapeutic purposes and procedures. Novel and innovative nanoparticles and nanodevices are expected to be exploited with an immense positive impact on human health. Over the next ten to twenty years nanotechnology may fundamentally transform science, technology, and society offering a significant opportunity to enhance human health in novel ways, especially by enabling early disease detection and diagnosis, as well as precise and effective therapy tailored to the patient. Nanomedicine could revolutionize the way we detect and treat damage to the human body and disease in the future, many techniques only imagined a few years ago are making remarkable progress towards becoming realities!

\section{Acknowledgements}

None.

\section{Conflicts of interest}

The authors declare that there are no conflicts of interest.

\section{Funding}

None.

\section{References}

1. Sangeeth kumar S. Applications of nanotechnology. KCT Coimbatore, India, 12MCA079. 2012.

2. http://www. academia. edu/5 124561 /Nanotechnolog yama3 112011111105104844-phpapp01

3. http://www.nano.gov/NNI-Manufacturing-at-the-Nanoscale.pdf.

4. Hood E. Nanotechnology: Looking as we leap. Environment Health Perspect. 2004;112(13):A740-A749.

5. nisenet.org/catalog/media/how_small_nano_video

6. www.slideshare.net/aniket272470/nanomedicine-presentation

7. www.nano.gov/nanotech-101/special

8. www.nano.gov/nanotech-101/special

9. www.oecd.org/dataoecd/37/19/37770473.pdf

10. www.gsjournal.net/old/science/manzelli.pdf

11. www.slideshare.net/Hammad37/nanomedicine-27647478

12. Syed Abeer. Future Medicine: Nanomedicine. JIMSA. 2012;25(3):188.

13. Sanjeeb K Sahoo. Applications of Nanomedicine. APBN. 2005;9(20):1048-1050.

14. www.slideshare.net/bhavithd/nanomedicine-5510006 and www. slideshare.net/.../an-overview-of-nanotechnology-in-medicine-361

15. Ankit Agarwal.The future of anaesthesiology. Indian $J$ Anaesth. 2012;56(6):524-528.

16. Jain KK. Nanomedicine: Application of Nanobiotechnology in Medical Practice. Med Princ Pract. 2008;17(2):89-101.

17. www.slideshare.net/kirtisingh2011/nanotechnology-ppt, www.unitar. org. 